\title{
Optics curriculum for middle school students
}

\section{Michael Nofziger}

Michael J. Nofziger, "Optics curriculum for middle school students," Proc. SPIE 2525, 1995 International Conference on Education in Optics, (13 October 1995); doi: 10.1117/12.224064

SDIE Event: SPIE's 1995 International Symposium on Optical Science, Engineering, and Instrumentation, 1995, San Diego, CA, United States 


\title{
Optics curriculum for middle school students
}

\author{
Michael Nofziger \\ Optical Sciences Center \\ University of Arizona \\ Tucson, Arizona 85721 \\ Electronic Mail: nofziger@gas.uug.arizona.edu
}

\begin{abstract}
A two-week course in optics has been developed and taught as part of the APEX program at the University of Arizona. APEX, (Academic Preparation for EXcellence), is an early-outreach program for minority students in the middle schools of the greater Tucson area. APEX targets financially disadvantaged students who possess skills necessary to be successful in higher education beyond high school. The program promotes academic confidence and career awareness through school-year meetings, field trips, a speakers' bureau, and a summer day camp. The optics course described in this paper has been taught over the last four years to middle school students in the APEX summer camp.

The optics course presented is a micro-version of the optics courses taught as part of the B.S. program in Optical Engineering at the University of Arizona. The APEX optics class contains a successful mixture of theory and hands-on demonstrations. Proof of this success is evident at the end of every summer session when, after two weeks and forty hours of optics, the students are eager to learn even more about the world of optics!
\end{abstract}

Keywords: optics education, middle school, demonstrations, curriculum planning

\section{INTRODUCTION}

The University of Arizona sponsors an educational outreach program entitled Academic Preparation for EXcellence (APEX). APEX targets middle and high school students who are minorities, financially disadvantaged, and/or whose parents never attended college. The program is designed to reach middle school students, and track them until they hopefully are accepted into college. It should be noted that a student must also maintain a certain GPA in order to qualify for the APEX program.

The APEX program is administered by having an outreach coordinator in most of the local middle and high schools in the southern Arizona area. This coordinator is typically a teacher or counselor in that particular school. He or she helps organize monthly APEX meetings and special events, including guest speakers from local businesses and professions. The students in general treat APEX with a sense of pride--it represents a level of success and achievement which they hope to accomplish through higher education.

An important aspect of APEX is a two-week summer program held on campus at the University of Arizona. Students may elect to study a particular scientific or engineering discipline, including optics, as part of this program. The classes meet every morning for two hours, with a separate study time in the afternoon. We have taught optics as part of this summer program for four years, this summer marking our fifth year. Each class averages about 20 students, the majority being from middle schools. We have had great success and have 
received enthusiastic praise from students, parents, and teachers alike in teaching the following optics curriculum.

\section{CURRICULUM}

The curriculum we follow is modeled after our undergraduate program in Optical Engineering. An introduction to the world of optics is first given, hinting to the students what lies ahead. The nature of light is then introduced, both as a wave and particle phenomena. Properties of waves are covered, including the concepts of velocity, frequency, and wavelength. The astronomical concept of a light-year is introduced (concepts from astronomy invariably crop up in an optics class such as this, and students always seem eager to learn about astronomy, too). At this time the entire electromagnetic spectrum is talked about, in order to put the visible spectrum in its perspective.

Geometrical optics is next introduced, including the laws of reflection and refraction (a challenge when the students have not yet been exposed to concepts in trigonometry). Flat mirrors are discussed and used. Prisms are then explained, demonstrating their use to either deviate or refract a beam of light. At this point the concept of total internal reflection is introduced, and the extension is made to explain how optical fibers work. The concept of a lens is then introduced, modelled simply as a stack of prisms. The concept of focal length is covered with reference to a collimated source. If enough of the students have had beginning algebra, the imaging equation may be introduced at this point. This allows the use of a simple lens bench to become a quantitative exercise vs. a pure qualitative one. Keplerian and Galilean telescopes are discussed, as are simple microscopes and binoculars. The optics of the human eye are covered in basic detail.

Sources of light are talked about, to include natural as well as man-made. It is here that familiar examples abound to the student, yet most have never thought of the differences between, say, a tungsten and fluorescent bulb. It is at this time that lasers are introduced. Discussion includes the many types, shapes, and sizes of lasers as well as the colors of light that they emit. As expected, this topic is a favorite one and draws many comments and questions.

Detectors are introduced next, without any specific regard to solid state physics or quantum mechanics (ahhh, how enjoyable!). It is here that common examples may not be so obvious to the student, yet many are aware that more exotic devices such as infrared night vision scopes exist.

The subject of image processing is included, both because of its increasing importance and its visual appeal. It is guaranteed success to show students astronomical or medical images downloaded directly off the Internet, and recent images from the Hubble Space Telescope are always a great hit!

Concepts from physical optics are covered next. Experience has shown that although this area of optics is difficult to teach conceptually, everyday examples abound. The colors of insect wings, soap and oil films, and lens coatings are but a few examples. A simple description of what a diffraction grating is comes next, and the spectra of various light sources are talked about. Diffraction gratings are handed out to the students so they can investigate and look for different spectra on their own, at home in their own surroundings. Holography is introduced simply, and a variety of holograms are handed out for viewing.

The last two days of class culminate by having the students build a complete working optical voice link, using 
kits developed in-house. ${ }^{1}$ The transmitter consists of a microphone, an amplifier stage of gain, and a transistor modulating an LED source in an AM mode. The receiver consists of a phototransistor, two stages of gain, and a speaker. The units are built following instructions written in the classic style of Heathkit....step by step! Receivers are built first, to allow for some simple experimenting. The rectified signal of $120 \mathrm{~Hz}$ from fluorescent lights is easily heard and compared to light from the sun and a computer screen. Transmitters are built next and tested for modulation.

The link is first tested with the LED and phototransistor in close proximity. Finally, the two units are coupled using inexpensive Fresnel lenses, allowing the students to send their voices as far as their "optical alignment expertise" allows. In a practical sense, most students achieve distances of 40-50 feet, with some having had success at 150 feet or so. This final project is a great combination of concepts learned throughout the course, and leaves the students with a valuable feeling of success and achievement.

\section{DETAILED DESCRIPTION}

\subsection{Course outline}

\section{The World of Optics}

Discover the world of optics with the help of an optical engineer! Learn about reflection, refraction, interference and diffraction. Experiment with lenses, mirrors, lasers and detectors. Build an optical voice link, and transmit your voice over space. Come experience the world of optics and its high-tech wizardry!

DAY 1 --Introduction

--What is optics?

--Optics around us (input from the students)

--What optical scientists do

--Make a rainbow!

--What is light?

--Color

-white light from red, green, blue

-laser goggles, colors, and the rainbow

DAY 2 --Reflection

-blackboard demos of mirrors

-student experiments with mirrors

-student experiments with simple periscopes

-index of refraction

-total internal reflection

-how optical fibers work

-student experiments with optical fibers 
DAY 3 --Refraction

-blackboard demos of prisms

-how a rainbow forms

--Lenses

-how a lens works

-blackboard demonstration

-positive and negative lenses

-focal length

-how an image forms

-student experiments with lenses

-student experiments with magnifiers

DAY 4 --Fresnel lenses

--Mirrors

-mirrors in the funhouse

-the floating penny

--Telescopes

-students make their own telescopes

DAY 5 --Review

--Group oral quiz

--Microscopes

-how they work

-student experiments with microscopes

--Binoculars

-how they work

--Our eye

-how it works

-experiments with our eyes

DAY 6 --Sources of Light

-student input

-the sun

-flames

-tungsten bulbs

-fluorescent lights

-neon signs

-glo sticks

-fireflies

-LASERS! (demos)

DAY 7 --Detectors of Light

-how they work

-visible detectors

-infrared detectors 


\title{
-student experiments
}

-cameras

--Image Science

-space images from the Information Superhighway

-images from the Hubble Space Telescope

\author{
DAY 8 --Interference \\ -demos \\ --Diffraction \\ -demos \\ -experiment to measure the wavelength of light \\ --Holograms \\ -view a variety of white-light holograms \\ --Talk Over a Beam of Light \\ -introduction to the experiment \\ -basic electronics \\ -begin building the kits
}

DAY 9

--Talk Over a Beam of Light

-build the receivers

-student experiments to detect light

\section{DAY $10 \quad$--Talk Over a Beam of Light \\ -build the transmitters \\ -align and test the link \\ --Review \\ -talk over a beam of light! \\ --Conclusions}

\subsection{Teaching ideas}

This section contains teaching ideas and observations of student response, drawn from personal experience in teaching this curriculum.

\subsubsection{Day one}

An introduction to the world of optics is given by having the students themselves think about all of the sources and uses of light in the world around them. Traditional instruments such as telescopes, microscopes, binoculars, and rifle sights are already part of their optical way of thinking. However, it is often surprising how many of them already recognize some of the more exotic uses of optics, including lasers, CD players, supermarket scanners, and remote controls. Even so, it is this author's belief that students benefit the most from recognizing the sources of light and optical phenomena found in nature, things not always thought of as "high-tech." Here in the desert, our summer displays of lightning and rainbows can be breathtaking. These are noted, and a rainbow is formed across the wall, using a prism and flat mirror placed in front of the imaging lens of an overhead projector. The prism disperses the white light into a familiar spectrum, at the same time 
introducing enough astigmatism to form an unexpected upwardly-curved beam. The mirror inverts this, creating a rainbow having the correct spectral and spatial orientation. At this point you will have captured the students' attention and interest in a most colorful way.

A discussion of the nature of light follows. Light is talked about as being either a wave or a particle, but not both simultaneously. This duality, this apparent contradiction comes as a great surprise and with much fascination to the students. A dramatic effect is achieved when it is pointed out that even the greatest minds in science have not been able to resolve this apparent discrepancy. From the start, the students are given a chance to see that not all of science has been discovered or explained, that much is left for them perhaps to solve someday.

The nature of light is then extended to a discussion of the entire electromagnetic spectrum. It is pointed out that mathematically, light waves are just like radio and TV waves, yet in many ways they are very different. This gives the student a point of reference when discussing the concept of how fast a light wave oscillates. All are familiar with the numbers representing the frequency of their favorite radio station, yet few realize just what those numbers mean.

\subsubsection{Day two}

This marks the start of geometrical optics. The law of reflection is introduced, once it is clear that everyone understands the concept of an angle! (For many middle school students this is a new concept.) Reflection is demonstrated both with a computer program and with a commercial blackboard optics kit. ${ }^{2}$ The kit contains large plastic prisms and lenses which magnetically stick to a blackboard. A light source with a cylinder lens delivers an individual fan of light, or "ray," through the prisms or lenses. In a darkened room, one can clearly see the ray as it propagates, illustrating reflection from a mirrored surface. Actual mirrors are given to each student to investigate reflection on their own. It is a fun exercise to have them all write their name backwards and view it, first in one, then two reflections. An everyday experience of this occurs when looking at the front of an ambulance in reflection from a car's rear-view mirror. This explains why the word "AMBULANCE" is written backwards on the front of the vehicle, something most students have already seen but maybe not thought about.

Index of refraction is introduced next, so that total internal reflection (TIR) in an optical fiber may be explained. This also provides the chance to review basic concepts from day one. The optics kit is again used to demonstrate TIR in a plane parallel plate, and the extension is made to round optical fibers. At this point, each student receives their own plastic fiber to investigate its properties. They are encouraged to see if only certain colors of light will be transmitted, or if the fiber seems to work for all colors. Given that the plastic fiber is of low quality, a fun experiment is to shine a bright flashlight through each of the fibers, to observe leakage along the length of the fiber. While entertaining, it should be pointed out that this is undesired in high-quality fibers!

\subsubsection{Day three}

This is a continuation of geometrical optics, introducing the concept of refraction. This is a challenge, as students of this age have not yet had any trigonometry. Writing down Snell's Law is like writing in a totally foreign language! This is solved by ignoring the sine of the angles, in effect writing down a small-angle approximation to Snell's Law (although this isn't mentioned to the students). Another useful approach, 
involving no math, is to mention Fermat's Principle and the connection to Snell's Law. (Be somewhat prepared, however, as usually some student will ask why rays behave like this!). The law of refraction is then demonstrated using a computer simulation program and the blackboard optics kit. (The program draws lines from a point object to a point image, simultaneously and all at the same speed on the screen. One of the lines is always along the axis, and the others make various angles. Different surfaces are encountered between the object and image, including a perpendicular plane, tilted plane, spherical, and off-center spherical surface. At the surface, each line is "refracted" so that it heads toward the point image. The line in red, the one that is in fact obeying Snell's Law, reaches the point image first, every time.....just like Fermat said!).

Once the concept of refraction is taught, prisms are introduced. To keep things simple, only equilateral and right-angle prisms are talked about. The equilateral prism is used to refract light, dispersing it into the rainbow spectrum. The right-angle prism is used to deviate a beam $90^{\circ}$ with no dispersion, as well as to demonstrate TIR. Both prisms are demonstrated using the blackboard optics kit.

The study of lenses is considered next. A lens is modelled simply as two stacks of "truncated" equilateral prisms, with a plane-parallel plate in the center. The prisms have increasing apex angles, so the outer prisms have a sharp pointed apex. This hypothetical stack of glass blocks is then "polished" into two smooth biconvex surfaces which form a positive lens.

At this point the shapes of lenses are drawn on the board, to include biconvex, plano-convex, biconcave, and plano-concave forms. A positive lens is defined as one that is thicker in the middle than at the edges. A negative lens is defined as one that is thinner in the middle than at the edges. Students are each given a set of 10 surplus lenses, (5 positive and 5 negative lenses), of various shapes. They are to sort the lenses according to whether they are positive or negative, based on their shapes. An advantage of using surplus stock is that no two sets contain the same lenses, which challenges the students to think on their own.

Once the lenses have been sorted, students are encouraged to pick them up and look through them, describing what they see. All of the negative lenses will of course produce an upright, demagnified virtual image. The positive lenses, depending on how they are used, will either produce an upright, magnified virtual image, or an inverted, magnified or demagnified real image (which in turn is viewed by the eye). Students invariably ask why a positive lens can produce either an upright or inverted image, while the negative lens only forms an inverted image. This is a difficult question to answer without showing simple ray tracing!

The concept of focal length is finally introduced, by showing a source at infinity producing parallel rays (interestingly, no student ever seems to ask about off-axis rays, namely the chief ray). Most students at this level recognize the fact that these are parallel rays, for which the word "collimated" is given. Using the prism model of a lens just described, the rays are shown to bend through steeper angles in going from the center to the outer edge of the lens. All are shown to converge to a single point, without regard to aberrations. The distance from the center of the lens to this focal point is labelled the focal length. Students are then asked to measure the focal lengths of each of their positive lenses. A white wall near an outside glass door provides a convenient spot to form colorful inverted real images of the outside world. Seeing cars and bicycles move upside down always brings a sense of excitement to the students! (This could also be done outside, with care taken to shield the image screen from direct sunlight.)

In all of these exercises using lenses, it is important to make sure that certain misconceptions are not 
introduced. ${ }^{3}$ Due to the random nature of the surplus lenses provided, there is no predetermined relationship between the diameters and focal lengths. In other words, one student's large diameter lens may have a longer focal length than another student's smaller diameter lens. The seemingly obvious (but erroneous) conclusion is that larger diameter lenses produce larger images. Each student should understand that image size is related to focal length only, while image brightness depends both on focal length and lens diameter.

\subsubsection{Day four}

To close out the discussion of lenses, a Fresnel lens is introduced. This is a perfect tie-in between prisms and biconvex lenses. Plastic Fresnel lenses are handed out, and again the focal length is measured. The subject of Fresnel lenses affords a chance to review concepts of prisms and lenses from the previous two days, a technique very useful for the students.

The next part of the class covers curved mirrors. These are not introduced along with plane mirrors, but saved until lenses are discussed. The concepts of focal length and center of curvature are a natural follow-on to the topic of lenses. Both concave and convex mirrors are talked about, and examples of each are shown. It is noted that the side mirror on most cars is a convex mirror, which produces a demagnified, upright image, just like a negative lens. Concave mirrors are described as the type used as the primary mirror in telescopes. The students are given concave mirrors, and are left to discover the effect when their eye is placed at the center of curvature! A commercial optical device known as the Mirage is shown next. This device uses two parabolic mirrors placed rim-to-rim, facing each other, with a hole cut out in the top one. They work to form a real image just above the hole, of whatever object is placed on the surface of the bottom mirror. A penny placed on the bottom mirror appears to "float" just above the hole, appearing very real yet not actually there.

The major part of this day's discussion is to introduce telescopes. To simplify the discussion, lenses are used to describe the workings of a telescope. Both Keplerian (two positive lenses) and Galilean (a positive objective and a negative eyepiece) telescopes are talked about. Each type may be drawn on the board, showing collimated marginal rays. The term afocal may be introduced, and it is important to point out that the observer's eye is what finally brings the rays to focus to a real image. To actually make a telescope, the lenses have to be positioned a distance apart equal to the sum of their focal lengths. The students are given meter sticks and clay, and are asked to make both types of telescope. With some care, these may be taken outside the classroom and used to look at some distant object.

\subsubsection{Day five}

This day begins with an oral quiz or review. It is usually more effective in a summer course to verbally ask the questions than it is to hand out a written test. The goal is to make the review a chance to bolster their confidence in their understanding of optics--not to point out all of the things they don't understand.

A variety of optical instruments are shown and talked about. The idea is that by understanding the basics of lenses, the student can now appreciate more complex optical instruments, without the complexities of ray tracing. Simple compound microscopes are described, and small pocket microscopes are used by the students to look at a variety of objects. One favorite is to look at a color half-tone print, to discover that an apparent color is in fact made up of a combination of dots of the complimentary colors! Next, binoculars are described, to show how prisms are sometimes used in a real instrument. Finally, the optics of the human eye are covered 
in basic detail. Optical illusions may be used to point out that our visual system certainly includes the brain, as well as the eye.

\subsubsection{Day six}

An entire day is devoted to studying sources of light. It is instructive to allow the students to think of various sources of light, instead of just providing them with a list. This is a good opportunity to point out the differences between tungsten and fluorescent bulbs, both in how they are constructed and the nature of the light they each emit. No discussion of sources would be complete without mentioning the word laser. Students at this age love to talk about lasers, and love to hear about big, powerful lasers! Small He-Ne lasers and diode laser pointers are demonstrated and compared to the light from a flashlight. To some extent, this discussion of lasers (and the actual use of one) is purposely saved until mid-way through the course, to help hold the students' attention during the more tedious discourse on lenses and mirrors. (They know from day one that they will get to see a laser operate, but they must first learn some of the fundamentals of optics.)

\subsubsection{Day seven}

This day is devoted entirely to a discussion of optical detectors of light and computer images. Most students have probably not used or given much thought to detectors (as compared to sources), yet many are familiar with night-vision systems (from television coverage of the Gulf War). The overall discussion of detectors may be broken down into imaging vs. non-imaging types.

It is important to point out that the most sophisticated of imaging detectors is our own human eye, with all of its built-in control and processing functions. At this point, the camera is introduced and talked about, including the fact that it forms a real but inverted image on the film. This is also the time to discuss modern-day video cameras and the basics of CCD chip technology.

A simple infrared source that most are familiar with is the TV or VCR remote control, with the detector being built into the TV or VCR. If one is available, it is fun to experiment by reflecting the beam off various surfaces (including mirrors) to see which ones scatter or reflect enough light to be sensed by the detector. Have the students experiment in their own homes and report their findings!

Another aspect of optics which is fascinating to middle school students is the world of computers, images, and image processing. If the equipment is available, point your Internet World Wide Web browser to the following address to view one of the world's largest collection of space images:

\section{http://seds.lpl.arizona.edu}

Also, a variety of freeware and shareware image processing programs are available. If hardware and time permit, students very much enjoy processing an actual image from, say, the Hubble Space Telescope!

\subsubsection{Day eight}

This day is reserved for a brief introduction to the world of physical optics. Lasers are used to demonstrate interference and diffraction from a variety of apertures. The diffraction grating is introduced in a simple 
manner, and is then used by the students, along with a meter stick, to measure the wavelength of light. This is a good exercise in that it allows the student to use macroscopic tools to measure a very small number. The concept of wavelength was discussed early-on in the course, so this also provides a good review and a sense of closure to that earlier topic.

The subject of holography is introduced, at least in a simple manner. The manner in which transmission holograms are made is described, along with the conditions for playback. A variety of holograms are shown to the students, including laser transmission and white light types.

Finally, the optical voice link kits are described and introduced. These are transmitter and receiver circuits that allow the students to send their voices over a beam of light. (See the reference for specific details about the kits.) The students are always excited about starting this part of the class. The opportunity for them to build circuits with their own hands is a great way to end the class on a high note of enthusiasm! On this day, the very basics of electronics needed for the kits are covered. The concepts of voltage, current, resistance, and Ohm's Law are talked about in very simple terms. In addition, the parts they will use are described, including resistors, capacitors, a transistor, and a couple of integrated circuits. The transistor and integrated circuits are talked about in general terms, treated as "building blocks." The goal isn't to have them become electrical engineers, but to become somewhat familiar with the parts they will use. In this way, the kits become something more than just "black boxes" full of mysterious parts.

\subsubsection{Day nine}

The receiver kits are handed out, along with the instruction manuals. The first step is to identify all of the parts, just like the Heathkit manuals of old (just like Heathkit's first step of identifying ALL parts, a step which many of us enjoyed skipping over!). The students are paired into groups of two, and quickly set to work. It usually becomes an unspoken race to see who can get their receiver to work first! It is very useful to troubleshoot the built kits by having a working copy of the receiver in-hand. This reduces looking at a schematic to just comparing their wiring with yours. In this way, errors are found with ease and pointed out to the students to fix.

Once a kit has passed "inspection" the detectors and speakers are handed out and the fun begins. The first source of light is usually from overhead fluorescent bulbs, which produce a $120 \mathrm{~Hz}$ buzzing sound from the speaker. The loudness varies with the amount of light falling upon the detector. (An interesting comparison is to "listen" to light detected from tungsten bulbs. The modulation depth of the light is nowhere near as great as it is with the fluorescents, making for a much quieter sound. This often comes as a surprise to even our undergraduate students, who are of course asked to provide the explanation for this difference!) Students are then encouraged to find other sources of light to "listen" to, including the sun, LED's, computer monitors, and TV's. A word of warning should be given here. Don't allow the speakers to come close to large-size monitors--the permanent magnet in the speaker will magnetize the mask at the front of the screen, distorting the color balance (hence the need for magnetically-shielded speakers in surround-sound systems). Unfortunate experience has shown this to be a "permanent" accident.

\subsubsection{Day ten}

This day is a continuation of the last one, except that the transmitters are built. Again, have a working unit on- 
hand with which to troubleshoot. Once the transmitters have "passed inspection," the LED's and microphones are handed out. In a matter of seconds the room will be alive with excited students as they discover that they can send their voices from LED to detector, if only over a distance of an inch or so. The final step is to use large Fresnel lenses to couple the two units together. Before the lenses can be used, the students must measure their focal length, as they are used to collimate the LED and to focus the light onto the detector, in an afocal manner. Have the students demonstrate their "optical alignment abilities" over distances of 5 to 10 feet first. This gives them the skill and confidence to tackle distances approaching 150 feet or more!

\section{RANDOM THOUGHTS}

Students of today, even middle school students, have a tremendous variety of stimuli input from the world around them. They live in a world of multi-channel digital satellite TV systems, blazing-fast multi-media computer hardware, CCD video cameras, and personal CD players. Many have all of these in their own homes. These items are sophisticated, flashy, and relatively easy to use. Contrast this to a typical science experiment-the hardware is often simplistic, outdated, confusing to use, and produces a result which may not be very interesting. It might therefore be easy and tempting to argue that teaching science in today's world is difficult, at best. Students perhaps have too many competing influences to be attracted to and impressed by a simple science experiment (or a two-week course in optics).

This author would make the claim that, in fact, the opposite may often be the case. Experience has proven that the simplest of demonstrations often produce the greatest interest and excitement from students (at least for grades 4-9). As any teacher will attest to, one of the greatest joys of teaching is having students unexpectedly show genuine enthusiasm for a simple experiment. (On the contrary, one of the most frustrating aspects of teaching is working hard to set up a more elaborate demonstration, only to have little interest shown from students.) The hypothesis put forth here is that students have gotten so used to living in a "high-tech" world that the simple demonstrations or experiments, in fact, best grab their attention and interest.

The world of optics contains a wonderful collection of such simple experiments and phenomena. By its nature, optics is a colorful and visually appealing science, with numerous such examples from the natural world around us. (The same could be said about many, if not most, of the physical sciences, although optics seems to have a greater advantage.)

A few examples may be useful. The rainbow is something most students, if not all, have observed while looking at a stormy sky. It is a colorful, somewhat mysterious and magical sight to see. Showing one in the classroom carries much of this same intrigue. The demonstration of how an optical fiber carries light from one end to the other always brings an excited response from students. At one recent demonstration to a large group of fourth graders, someone wanted to know what would happen if light of different colors travelled down the fiber in opposite directions. The light sources were already set up, an impromptu experiment was carried out, and the students were, to put it mildly, thrilled! To the seasoned optical scientist, the result was trivial---there was no "mixing" of the colors. To the fourth graders, it was a monumental and amazing discovery! Another example involves the use of the blackboard optics kit described in this paper. In a darkened room, one can clearly see the "ray" as it propagates, illustrating reflection, total internal reflection, refraction, and dispersion. Again, to the seasoned optical scientist, the effect is expected and ordinary. From students, it always brings a unanimous response of delight and amazement. Finally, one favorite example is having the students use a positive lens to form an image. This simple demonstration always seems to instill in them a sense of discovery 
and satisfaction. Interestingly, many students know already know that the human eye forms an inverted image on the retina. Most, however, have never actually used a lens to form such an image.

\section{CONCLUSIONS}

The world of optics affords a unique and excellent opportunity for middle school teachers and optics professionals alike to reach out and affect the lives of our young students of today. Through basic teaching and demonstrations of optics, students gain insight into more complex instruments and products in the world around them. Our lives are becoming increasingly affected by optics--time put into teaching optics to our future generations is time well worth spending!

\section{REFERENCES}

1. M.J. Nofziger, "'Talking over a beam of light!': electro-optics suitable for K-12," Proc. SPIE $\underline{2525}$, paper no. 21 (1995).

2. This particular kit was sold by Klinger Educational Products as part number KO4100.

3. S.M. Pompea and M.J. Nofziger, "Resources for optics in middle school education," Proc. SPIE $\underline{2525}$, paper no. 20 (1995). 